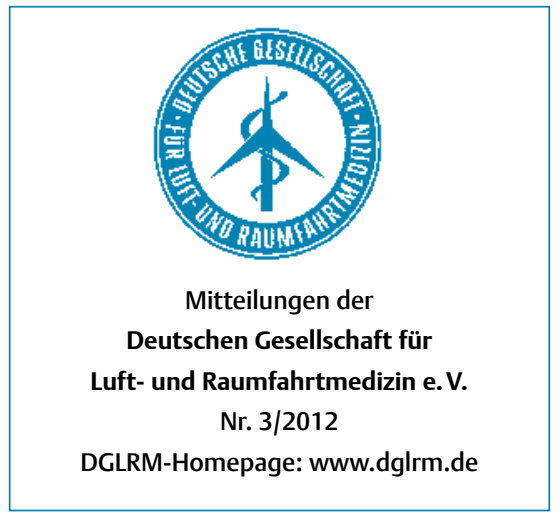

Geschäftsstelle der DGLRM

Frau Doris Mittelstädt - DLR

Linder Höhe

51170 Köln

E-Mail: geschaeftsstelle@dglrm.de

Sprechzeiten: mittwochs 14:00-16:00 Uhr

Tel.: 0176/56242876
Verantwortlich für den Inhalt der DGLRM-Seiten

Deutsche Gesellschaft für Luft- und

Raumfahrtmedizin e. V. (DGLRM)

Dr. Claudia Stern, Präsidentin, DLR,

51170 Köln, claudia.stern@dlr.de

Redaktionsschluss: 04.06.2012

Bankverbindung

Deutsche Bank Hamburg,

Konto-Nr.: 3889482, BLZ: 20070000

IBAN: DE 63200700240388948200 ,

SWIFT/BIC: DEUTDEDBHAM

Änderungen für die Mitgliederkartei bitte an: Dr. Martin Trammer, DLR,

51170 Köln, martin.trammer@dlr.de

Wir begrüßen als neues Mitglied

Dr. Udo Bonjean, Bad Tölz

Wir gratulieren

- 65 Jahre, Dr. Siegbert Müller, Bruchsal, 11.06.1947

- 70 Jahre, Dr. Klaus Reichenbach-Klinke, Fürstenfeldbruck, 21.05.1942

- 75 Jahre, Dr. Oberst a. D. Dietrich Oswald Wirth, Dresden, 21.06.1937

- 80 Jahre, Dr. Jürgen Kressin, Berlin, 15.06.1932

\title{
Liebe Mitglieder der DGLRM,
}

die Tagung der Aerospace Medical Association fand in diesem Jahr in Atlanta statt. Erstmalig gab es mehr Anmeldungen aus dem Ausland als aus den USA. An der traditionellen Sitzung der DGLRM Montagmittag nahmen circa 30 Personen teil, auch aus dem Ausland. In dieser Sitzung berichteten wir über die Tätigkeiten der DGLRM im letzten Jahr und diskutierten den Entwurf der neuen Satzung. Die Satzungs-AG der DGLRM, bestehend aus Eckard Glaser, Dr. Jörg Hedtmann, PD Dr. Carla Ledderhos, Dr. Matthias von Mülmann und Prof. Dr. Hans Pongratz hat viel Arbeit und Zeit in diesen Entwurf investiert, der den Mitgliedern mit der Einladung zur Mitgliederversammlung zugesendet werden wird. Am Mittwochnachmittag fand die deutschsprachige Sitzung statt, die Prof. Pongratz auch in diesem Jahr wieder organisierte. Das Thema des Panels lautete "Civilian and military disabled pilots: Preventive, diagnostic and therapeutic strategies in Germany“. Die Themen umfassten neben der kognitiven Beeinträchtigung des alternden Piloten, den behinderten Piloten aus orthopädischer Sicht, Hörhilfen bei Flugbesatzungen, das derzeitige Wissen um positive Druckbeatmung in Anti-G-Systemen sowie das BrugadaSyndrom.

Im März hatte ich das große Vergnügen an der 18. Fliegerärztliche Fortbildung in St. Auban teilzunehmen. An 12 Tage fanden vormittags flugmedizinische Vorträge und nachmittags praktische Übungen statt. Die praktischen Übungen im Rahmen von Segelflügen in einem der schönsten europäischen Segelfluggebiete sind für viele Teilnehmer unvergessliche Erlebnisse, die einen großen Beitrag zur Flugerfahrung und Berufserfahrung als Fliegerarzt leisten. Eine engere und bessere Verknüpfung von Theorie und Praxis ist kaum vorstellbar. 26 Referenten, unter ihnen viele DGLRM-Mitglieder, der deutsche Weltmeister im Segelflug Bruno Gantenbrink, der deutsche As- tronaut Dr. Ulf Merbold, die AMC-Leiter Prof. Dr. Uwe Stüben und Götz Kluge sowie Kollegen und Kolleginnen aus den Fachdisziplinen Kardiologie, Innere, Chirurgie, Gastroenterologie, Urologie und Ophthalmologie gestalteten das Vortragsprogramm. Den Organisatoren Dr. Martin Gräf, Peter Ross, Dr. Heiko Wassill und allen voran Jürgen Knüppel ein herzliches Dankeschön für ihr Engagement und die hervorragende Fortbildung der DGLRM.

Die Deutsche Gesellschaft für Luft- und Raumfahrt feiert 2012 ihr 100-jähriges Jubiläum. Aus diesem Anlass fand im April in der Landesvertretung NordrheinWestfalen in Berlin ein Festakt statt, zu dem ich eingeladen war. Unter den Festrednern befanden sich der Koordinator der Bundesregierung für Luft- und Raumfahrt, Parlamentarischer Staatssekretär Peter Hintze, Dr. Thomas Enders, Präsident des Bundesverbands der Deutschen Luft- und Raumfahrtindustrie (BDLI) e.V., und der Vorstandsvorsitzende des Deutschen Zentrums für Luft- und Raumfahrt (DLR) e.V., Prof. Dr. JohannDietrich Wörner. Unter den 230 Gästen befanden sich auch Sigmund Jähn und Hartmut Sänger. Wir gratulieren der DGLR zur ihrem 100-jährigen Bestehen ganz herzlich.

Ich wünsche Ihnen einen schönen Sommer und freue mich, Sie im September in Bonn wiederzusehen.

Claudia Stern
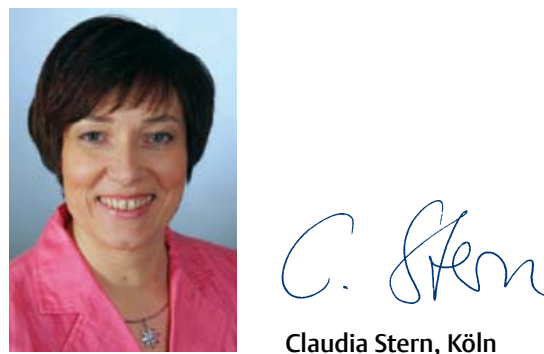

Claudia Stern, Köln 


\section{Rainer Kowoll Preis}

Wie bereits angekündigt, wird auf der bevorstehenden 50. Jahrestagung der DGLRM in Bonn erstmalig ein FirstPresenter-Preis vergeben. Dieser soll gemäß Vorstandsbeschluss „Rainer Kowoll Preis“ heißen. Die wissenschaftliche Schaffenskraft von Dr. Rainer Kowoll, ein hervorragender Jung-Wissenschaftler, ist das Vorbild für die zukünftigen Preisträger.

Kowoll war Sanitätsoffizier der Bundeswehr. Nach mehrjähriger Tätigkeit als Fliegerarzt in der Abteilung Flugphysiologie des Flugmedizinischen Instituts der Luftwaffe in Königsbrück wurde er als wissenschaftlicher Mitarbeiter an das Zentrum für Weltraummedizin (ZWMB) der Charité in Berlin abgeordnet. Hier forschte er im Bereich der Flug- und Höhenmedizin.

Für seine hervorragenden wissenschaftlichen Arbeiten wurde er unter anderem mit dem Förderpreis Hans-Hartwig-Clasen, dem Förderpreis der Liselotte und Dr. Karl Otto Winkler Stiftung sowie im Jahr 2005 auch mit dem wissenschaftlichen Förderungspreis der Österreichischen Gesellschaft für Alpinund Höhenmedizin (ÖGAHM) ausgezeichnet.

Im Juli 2006 verstarb Dr. Rainer Kowoll im Alter von erst 38 Jahren in Berlin.

\section{Basic-Life-Support (BLS) und Advanced-Life-Support (ALS)}

Im Rahmen unserer Jahrestagung bieten wir auch dieses Jahr wieder praxisorientierte Kurse aus dem Bereich der Notfallmedizin an. Die Workshops werden am Freitag, den 28. September, als Parallelveranstaltung zu unserem Vortragsprogramm stattfinden.

Am Vormittag sind ein bis zwei Workshops von jeweils 90 Minuten zu den Basismaßnahmen der Reanimation (BLS, gemäß aktueller ERCGuideline) mit maximal je 12 Teilnehmern/Kurs geplant. Am Nachmittag soll es darauf aufbauend einen Workshop Advanced-Life-Support (ALS) geben. Dieser Workshop wird auch allen Interessenten mit Erfahrung im Bereich der Notfallmedizin empfohlen.

Ihre Anmeldung richten Sie bitte direkt an die Kongressorganisation (dglrm2012@m4-cp.de). Für die Workshops wird ein Unkostenbeitrag von 35 Euro/Teilnehmer erhoben. Die Zertifizierung durch die Ärztekammer wird beantragt. Die Vergabe der Kursplätze erfolgt nach Eingang der Anmeldungen.

Wir möchten gleichzeitig alle Interessierten noch einmal herzlich zur 50. Jahrestagung der DGLRM im September in Bonn einladen. Bei Eingang Ihrer Anmeldung bis zum 30. Juni können Sie hierbei noch von vergünstigten Teilnahmegebühren profitieren. Weitere Informationen zu der Tagung finden Sie auch auf unserer Homepage (www.dglrm.de).
Kongresse 2012

Symposium DGVM/DGVP 2012

Dt. Ges. f. Verkehrsmedizin und

Dt. Ges. f. Verkehrspsychologie

Termin: 07.09.-08.09.2012

Ort: Hamburg

Kontakt: Frau Mandy Wagner, Tel.: +49 (0)3641/3116160

Internet: www.conventus.de/verkehr2012

$\mathbf{6 0}^{\text {th }}$ International Congress of Aviation and Space Medicine (ICASM)

Int. Academy of Aviation

and Space Medicine

Termin: 16.09.-20.09.2012

Ort: Melbourne, Australien

Internet: www.iaasm.org

\section{Jahrestagung DGLRM}

Dt. Ges. f. Luft- und Raumfahrtmedizin

Termin: 27.09.-29.09.2012

Ort: Bonn, Hotel Königshof

Internet: www.dglrm.de

$3^{\text {rd }}$ European Conference in Aerospace

Medicine (ECAM)

Termin: 09.11.-11.11.2012

Ort: $\quad$ London, Heathrow, Sheraton Skyline Hotel

Internet: www.ecam2012.eu

Fortbildungen 2012

29. Bayerische Fliegerärztetagung

Termin: 23.06.2012

Ort: Fürstenfeldbruck,

Flugmedizinisches Institut der

Luftwaffe (AMC)

Kontakt: fliegeraerztetagung@lvbayern. de

Flugmedizinischer Lehrgang Ic

(AME Klasse 1)

Termin: 16.07.-27.07.2012

Ort: Fürstenfeldbruck,

Flugmedizinisches Institut der

Luftwaffe (AMC)

Kontakt: Generalarzt der Luftwaffe, Luisenstr. 109, 53721 Siegburg, Tel.: +49 (0)2241/991683

EUSAM $-5^{\text {th }}$ International Aero Medical Examiner Seminar (FAA)

European School of Aviation Medicine

Termin: 26.08.-26.08.2012

Ort: Berlin, Hotel Palace

Kontakt: Frau Froese, Tel.: +49 (0)69/69691222

Internet: www.flugmed.org/ www.eusam.org
EUSAM - Basic course 22 (englisch) European School of Aviation Medicine Termin: 01.09.-09.09.2012

Ort: Frankfurt Airport, Lufthansa-Basis

Kontakt: Frau Froese, Tel.: +49 (0)69/69691222

Internet: www.flugmed.org/ www.eusam.org

DAF - Refresher-Seminar Flugmedizin (deutsch)

Dt. Akademie für Flug- und Reisemedizin

Termin: 26.10.-28.10.2012

Ort: Seeheim-Jugenheim, Lufthansa Training \& Conference Center

Kontakt: Frau Froese, Tel.: +49 (0)69/69691222

Internet: www.flugmed.org

EUSAM - Advanced course 22 (englisch) European School of Aviation Medicine Termin: 08.12.-16.12.2012

Ort: Frankfurt Airport, Lufthansa-Basis

Kontakt: Frau Froese, Tel.: +49 (0)69/69691222

Internet: www.flugmed.org/ www.eusam.org 


\title{
Deutsche Gesellschaft für Luft- und Raumfahrtmedizin e. V.
}

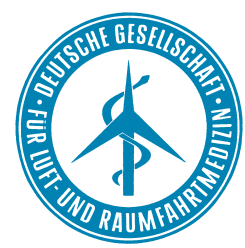

\section{Jahrestagung 27.-29.09.2012 im Hotel Königshof in Bonn}

\author{
Anmeldung \\ per Fax: +49 (0) 2286297331 \\ per Mail: dglrm2012@m4-cp.de
}

Kongressteilnahme 28.09. und 29.09.2012

Teilnahme an der Vorstandsrats-Sitzung am 27.09.2012

Teilnahme an den Fachbesichtigungen am 28.09.2012:

- Hubschrauber-Fliegergruppe der Bundespolizei mit $\square$ Personen oder

- ADAC HEMS Academy mit $\square$ Personen

Teilnahme am Festabend am 29.09.2012 mit $\square$ Personen

(Anmeldung bis 31.08.2012 erforderlich, Sie werden wegen der Sicherheitsüberprüfung durch das Bundespräsidialamt in einem separaten Anschreiben zur namentlichen Anmeldung aufgefordert

- Teilnehmerzahl begrenzt)

Titel, Name, Vorname

Institution / Firma

Anschrift

Telefon/Telefax

E-Mail (unbedingt erforderlich)

Teilnahmegebühren

Anmeldung bis 30.06.2012

Anmeldung ab 01.07.2012

Student mit Ausweis 40,-€

Mitglieder

$80,-€$

Mitglieder

$120,-€$

Nichtmitglieder

$120,-€$

Nichtmitglieder

$160,-€$

Fachbesichtigungen am 28.09.2012: $\quad 10,-€$

Festabend am 29.09.2012: $\quad$ 45,-€

$\square$ Mitglied der DGLRM $\square$ Nichtmitglied $\quad \square$ Student $\quad \square$ Ich habe Interesse an einer Mitgliedschaft

Ihre Anmeldung wird erst nach Eingang der Teilnahmegebühren gültig.

Hierzu steht Ihnen bei Angabe Ihrer Kontodaten

das Lastschriftverfahren zur Verfügung:

Bitte buchen Sie die Teilnahmegebühr von meinem Konto ab:

Kontonummer

BLZ

Bank

Kontoinhaber

Datum

Unterschrift
Alternativ können Sie die

Teilnahmegebühr überweisen an:

DGLRM e. V.

Kontonummer: 388948201

BLZ: 20070000

Deutsche Bank Hamburg

Verwendungszweck:

Jahrestagung 2012 